\title{
Polarization Optimization Studies for OPPIS
}

\section{Grigor Atoian'}

Brookhaven National Laboratory, Upton, NY, 11973, USA

E-mail: atoian@bnl.gov

\section{Victor Klenov}

Institute of Nuclear Researches, Moscow, Russia

E-mail: klenov@AL20.inr.troitsk.ru

\section{John Ritter}

Brookhaven National Laboratory, Upton, NY, 11973, USA

E-mail: jritter@bnl.gov

\section{Dannie Steski}

Brookhaven National Laboratory, Upton, NY, 11973, USA

E-mail: steski@bnl.gov

\section{Anatoli Zelenski}

Brookhaven National Laboratory, Upton, NY, 11973, USA

E-mail: zelenski@bnl.gov

\section{Valeri Zubets}

Institute of Nuclear Researches, Moscow, Russia

E-mail: vzubets@bnI.gov

Abstract

OPPIS (Optically Pumped Polarized Ion Source) $\mathrm{H}^{-}$ion source had been upgraded to a higher intensity and polarization. The depolarization factors in the multi-step spin-transfer polarization technique in the OPPIS with Fast Atomic Beam Source (FABS) source were studied. Optimization of the OPPIS parameters resulted in the OPPIS polarization increase to $84 \%$.

XVth International Workshop on Polarized Sources, Targets, and Polarimetry September 9-13, 2013

Charlottesville, Virginia, USA

1

\section{Grigor Atoian}




\section{Introduction}

The RHIC OPPIS had been upgraded for Run-2013 to higher intensity and polarization with a FABS $[1,2]$. The FABS source produces a high intensity and brightness primary proton beam. Part of this beam is converted to electron-spin polarized H-atoms by electron pick-up in an optically pumped $\mathrm{Rb}$ vapor cell (see Fig.1).

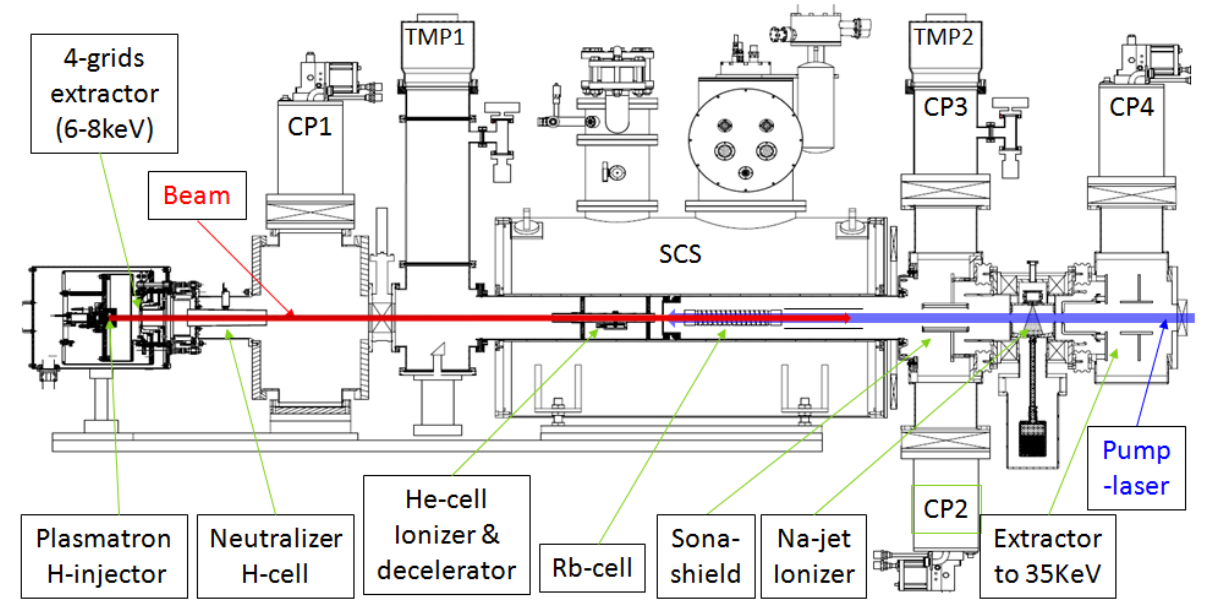

Figure 1: Optically Pumped Polarized $\mathrm{H}^{-}$ion Source

The new source layout is presented in Figure 1. The FABS consists of the plasmatron, the 4grids extractor and the hydrogen cell neutralizer. Hydrogen is injected into the plasmatron by a pulsed valve. The proton beam is produced by the 4-grid Ion Optical System and then it is neutralized in the H-cell neutralizer. A permanent magnet is placed downstream of the $\mathrm{H}$-cell to remove any residual $\mathrm{H}^{+}$or other charged species. The FABS produces the atomic hydrogen beam of equivalent intensity $\sim 3 \mathrm{~A}$ at beam energy of $6-8 \mathrm{keV}$. The atomic beam is injected inside the Superconducting Solenoid (SCS) where it is partially ionized to protons in the pulsed gaseous He-cell. The HV bias applied to the He-cell decelerates this proton to $3-4 \mathrm{keV}$ as shown in Fig.2. The energy of the non ionized part of the beam remains unchanged.

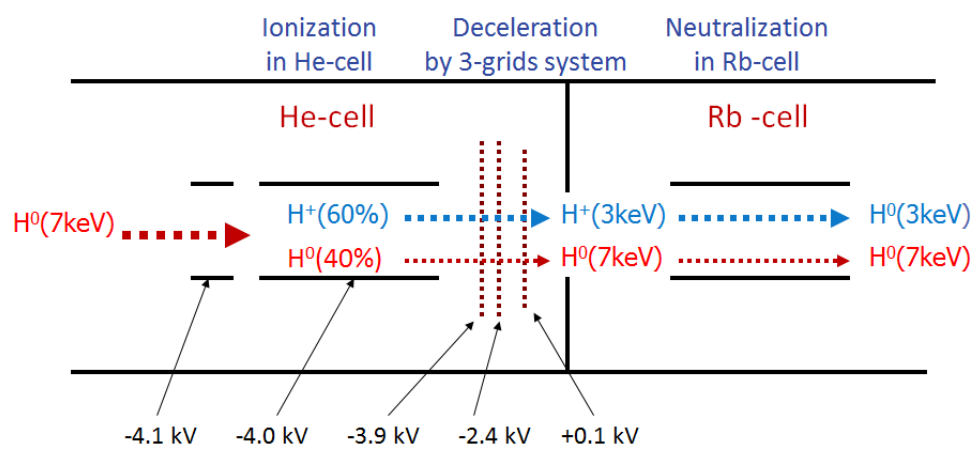

Figure 2: Energy separation system

This ionized beam picks up polarized electrons from the $\mathrm{Rb}$ atoms to become a neutral beam of electron-spin polarized $\mathrm{H}$ atoms. The deflector plates downstream of the $\mathrm{Rb}$-cell remove any residual charged species. Finally, we have a neutral beam that contains two components: one part is the $\mathrm{H}$ atoms with polarized electrons at about 3-4 $\mathrm{keV}$ energy and the 
other part is atomic $\mathrm{H}$ beam with unpolarized electrons at energy $6-8 \mathrm{keV}$. The electronpolarized $\mathrm{H}$-beam then passes through a magnetic field reversal region, where the polarization is transferred to the nucleus, via hyperfine interaction (Sona transition). The atoms are then negatively ionized and accelerated in a Na-jet vapor cell to form nuclear polarized and unpolarized $\mathrm{H}^{-}$ions. Further suppression of unpolarized components occurs in the LEBT (low energy beam transport) line and the RFQ. The unpolarized beam is well separated after a bending magnet in the LEBT. The system of collimators and the additional suppression in RFQ almost completely eliminates this component.

\section{The depolarization factors of OPPIS}

The OPPIS technique is a multi-step polarization-transfer process [3]:

- production of circular polarized light by a tunable wavelength $(\sim 795 \mathrm{~nm})$ beam laser;

- transfer of polarization from the laser beam to an electron in the $\mathrm{Rb}$ atoms by the optical pumping technique;

- production of an electron spin polarized $\mathrm{H}$ atoms by capture of a polarized electrons by protons from $\mathrm{Rb}$ atoms;

- polarization transfer from the electron to the proton by "Sona-transition" technique;

- ionization of the hydrogen atoms by the capture of a second electron in Na-jet for acceleration.

At each step there is some loss of polarization, which can be expressed as follows:

$$
\mathbf{P}=\mathbf{E}_{\mathrm{H} 2} \cdot \mathbf{P}_{\mathrm{Rb}} \cdot \mathbf{S} \cdot \mathbf{B}_{\mathrm{RG}} \cdot \mathbf{E}_{\mathrm{LS}} \cdot \mathbf{E}_{\mathrm{ES}} \cdot \mathbf{E}_{\mathrm{Sona}} \cdot \mathbf{E}_{\mathrm{ion}}
$$

2.1 Dilution due $\mathrm{H}_{2}{ }^{+}$in the new source $\left(\mathrm{E}_{\mathrm{H} 2}\right)$ The dilution of polarization due to the $\mathbf{H}_{2}{ }^{+}$ component is less than $1 \%$ [4]. The $\mathbf{H}_{2}{ }^{+}$component of the beam from the plasmatron is less than $10 \%$ and in the $\mathrm{H}$-cell of FABS it becomes neutral H-atoms with energy of 3-4 keV, which cannot be separated by the bending magnet and collimators. Additionally this beam is attenuated due to larger angular divergence. After the Na-ionizer this part of the current is 100 times less than the polarized $\mathrm{H}^{-}$ions current and the factor $\mathbf{E}_{\mathbf{H} 2} \sim 0.99$.

2.2 Rb-optical pumping $\left(\mathbf{P}_{\mathbf{R b}}\right)$ The beam polarization strongly depends on the power, the frequency, and the line width of the pumping laser. Monitoring and control of these parameters are critical to prevent polarization loss. An "Angstrom WS/6 200" wavelength meter was used to control the laser $\left(\mathbf{P}_{\mathbf{R b}} \sim 0.99\right)$.

2.3 Rb polarization spatial distribution (S) The spatial distribution of the laser spot and the proton beam represents the degree of matching between the $\mathrm{Rb}$ polarization profile and the spatial proton beam profile (Fig.4). The optimization of the beam size, the laser spot and the diameter of the $\mathrm{Rb}$-cell can improve the polarization (S 0.98).

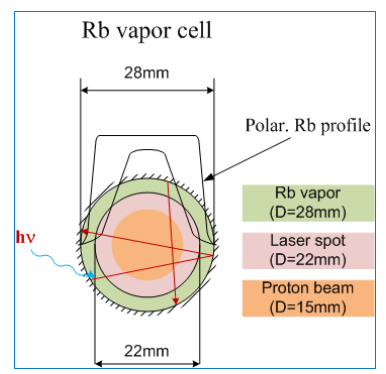

Figure 4: Spatial distribution of proton beam, vapor of $\mathrm{Rb}$ and laser spot at the $\mathrm{Rb}$-cell 
2.4 Proton neutralization in residual gas $\mathbf{B}_{\mathbf{R G}}$ The neutralization of the ionized beam, by the residual gas upstream and inside the $\mathrm{Rb}$-cell, produces unpolarized hydrogen atoms. This part of the beam can't be separated in LEBT, which dilutes the beam polarization. The factor $\mathbf{B}_{\mathbf{R G}}$ can be calculated from polarization at different thicknesses of Rb vapor (Fig.5).

The dilution of polarization due to residual gas at the operational $\mathrm{Rb}$ thickness of $\sim 2.5 * 10^{13}$ atoms $/ \mathrm{cm}^{2}$ is $\mathbf{B}_{\mathbf{R G}} \sim 0.985$. This polarization loss can be reduced by better vacuum pumping and optimization of the cells size.

2.5 Depolarization due to spin-orbital interaction $\left(\mathbf{E}_{\mathrm{LS}}\right)$ The curve in Fig.6 shows the polarization saturation in the magnetic field of the SCS above $25 \mathrm{kG}$.

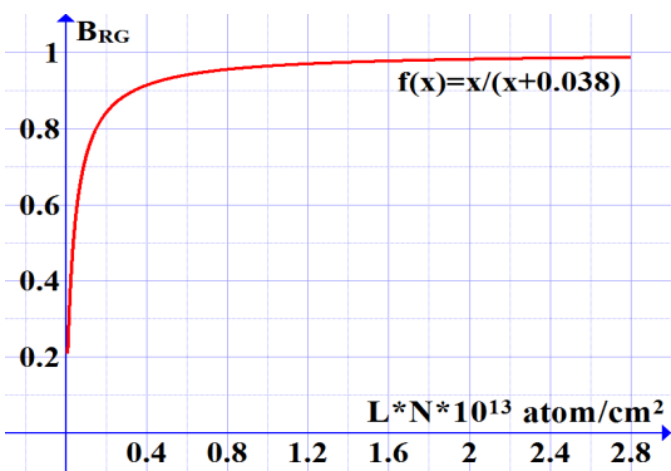

Figure 5: The factor of residual gas $\mathbf{B}_{\mathbf{R G}}$ vs. the thickness of $\mathrm{Rb}$

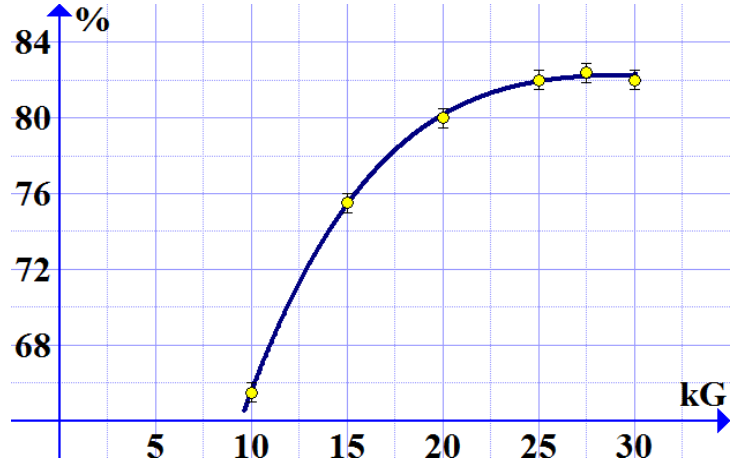

Figure 6: Dependence of polarization on the magnetic field of the SCS

2.6 Dilution due to incomplete energy separation of the unpolarized component of the beam $\left(\mathbf{E}_{\mathbf{E S}}\right)$ The ratio of the unpolarized part of the beam to the polarized after the Na-ionizer is about 0.25 [4]. The energy separation by the bending magnet and collimators is better than 25 30 times.

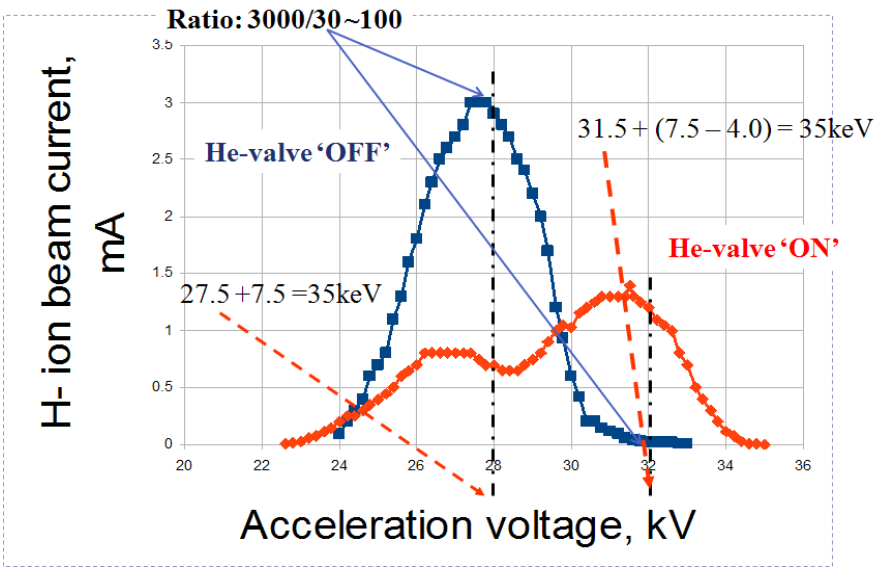

Figure 7: Current out of the LINAC with He-valve "OFF"(red) and "ON"(blue)

Finally the dilution of polarization can be reduced by the energy separation of the $\mathrm{H}^{-}$beam ( 25-30 times) to $\sim 0.01$ (Fig.7) and dilution due to incomplete energy separation $\mathbf{E}_{\mathbf{E S}} \sim 0.99$. 
2.7 Sona-transition efficiency ( $\mathbf{E}_{\text {Sona }}$ ) Five correction coils (LCC, SCC, ICC1, ICC2 and ICC3) are used for optimizing the magnetic field in the Sona-shield to achieve maximum polarization (Fig.8).

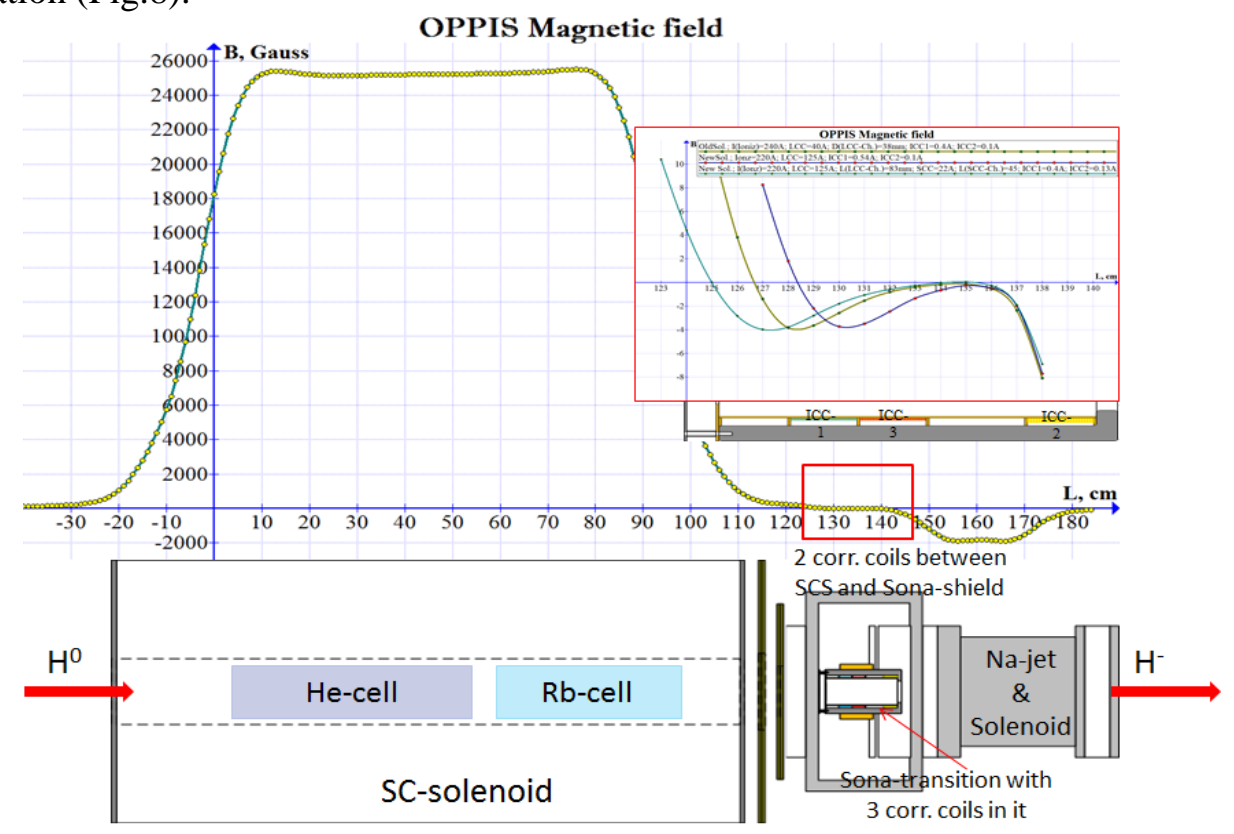

Figure 8: OPPIS magnetic field produced by the SC-solenoid, 5 correction coils, Sona-shield and Na-jet solenoid

For maximum polarization, all correction coils must be accurately set. Any change in the magnetic field of the correction coils, the SCS or the ionizer as well as their position requires new settings. Scans of the correction coils indicate that the Sona-transition efficiency is similar for the ECR and FABS based OPPIS.

2.8 Incomplete hyperfine interaction breaking in the ionizer magnetic field $\left(\mathbf{E}_{\mathrm{ion}}\right)$ To break the electron-proton hyperfine interaction a magnetic field strength of about $1.5 \mathrm{kG}$ is required in the sodium-jet ionizer cell. The factor $\mathbf{E}_{\text {ion }}$ is presently estimated at: $\mathbf{E}_{\text {ion }} \sim \mathbf{0 . 9 7 - 0 . 9 8}$ (for ionizer field of 1.5-2.0 kG). The benefits of the higher ionizer field for polarization increase are offset by current reduction due to emittance growth (Fig.9). A higher brightness polarized atomic $\mathrm{H}$ beam is required to keep beam emittance in the RFQ acceptance range at high field by using smaller diameter collimated beam.
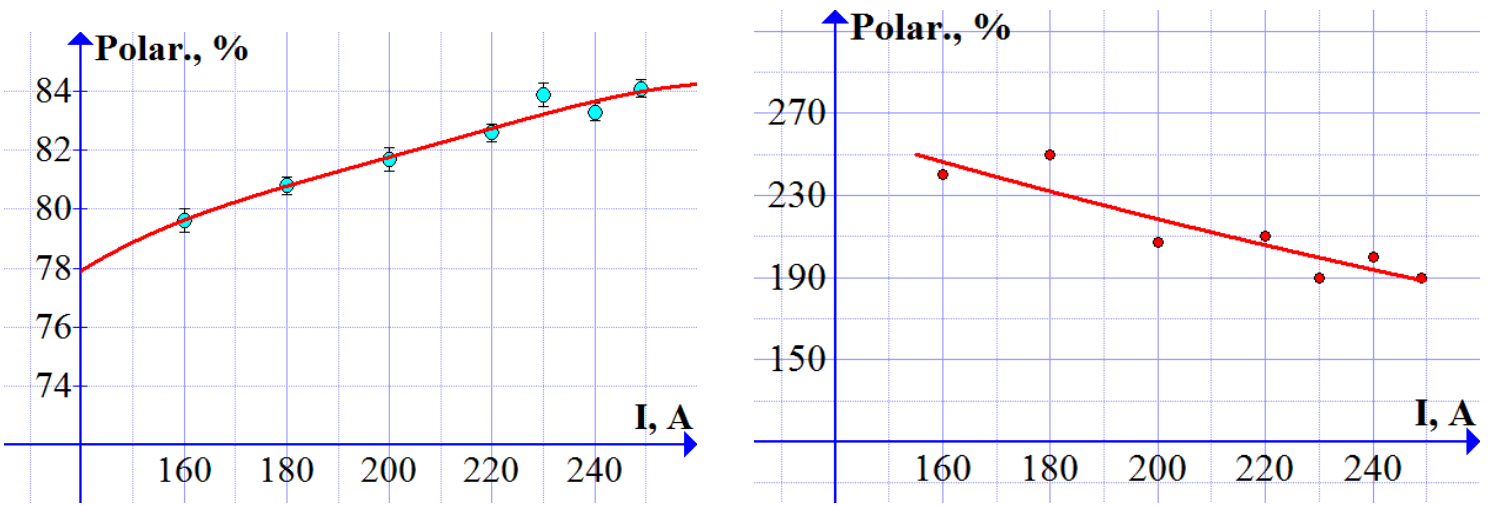

Figure 9: Polarization and current vs. the magnetic field of the Na-jet solenoid 


\section{Summary}

The depolarization factors for the ECR and FABS based OPPIS are compared in table 1 .

Table 1: The depolarization factors of OPPIS with the FABS source

\begin{tabular}{|l|l|l|c|c|}
\hline \multicolumn{2}{|c|}{ Depol. factor } & \multicolumn{1}{|c|}{ Process } & ECR-type & FABS-type \\
\hline 1 & $\mathrm{E}_{\mathrm{H} 2}$ & Dilution due $\mathrm{H}_{2}{ }^{+}$ & 0.98 & 0.99 \\
\hline 2 & $\mathrm{P}_{\mathrm{Rb}}$ & Rb-optical pumping & 0.98 & 0.99 \\
\hline 3 & $\mathrm{~S}$ & Rb polarization spatial distribution & 0.97 & 0.97 \\
\hline 4 & $\mathrm{~B}_{\mathrm{RG}}$ & Proton neutralization in the residual gas & 0.95 & 0.98 \\
\hline 5 & $\mathrm{E}_{\mathrm{LS}}$ & Depolarization due to spin-orbital interaction & 0.98 & 0.98 \\
\hline 6 & $\mathrm{E}_{\mathrm{ES}}$ & Dilution due to incomplete energy separation & 1.0 & 0.98 \\
\hline 7 & $\mathrm{E}_{\mathrm{Sona}}$ & Sona-transition efficiency & 0.96 & 0.96 \\
\hline 8 & $\mathrm{E}_{\text {ion }}$ & $\begin{array}{l}\text { Incomplete hyperfine interaction breaking in } \\
\text { the ionizer magnetic field }\end{array}$ & 0.98 & 0.98 \\
\hline & Total & & $\sim 81.5$ & $\sim 84.5$ \\
\hline
\end{tabular}

Polarization of the upgraded OPPIS is on average about 3-4\% higher than the old ECR-based source. It is expected that polarization can be further improved to $86 \%$. Higher polarization is expected due to reduced depolarization factors:

- $\quad \mathrm{Rb}$ polarization spatial distribution;

- reduce residual gas;

- Sona-transition efficiency;

- incomplete energy separation and

- Incomplete hyperfine interaction breaking in the ionizer magnetic field.

\section{References}

[1] A. Zelenski et al., "RHIC polarized source upgrade”, PAC-2011 conference, (2011)

[2] A. Zelenski, "Polarized source upgrade for RHIC", SPIN PHYSICS (SPIN2012) JINR, Dubna, Russia, (2012)

[3] A. Zelenski et al., "The RHIC optically-pumped polarized H ion source”, PSTP-2007 conference, BNL, USA, (2007)

[4] A. Zelenski et al., "The RHIC polarized source upgrade", Spin Physics Symposium (SPIN 2010) Juelich, Germany, (2010) 